\title{
The Effects of Motivational Self-Talk on Competitive Anxiety and Self-Compassion: A Brief Training Program among Competitive Swimmers
}

\author{
Styliani Kyriaki Georgakaki1,2, Eirini Karakasidou, ${ }^{1,2}$ \\ ${ }^{1}$ Institution of Counselling and Psychological Studies, I.C.P.S., Athens, Greece \\ ${ }^{2}$ University of Central Lancashire, Preston, UK \\ Email: skgeorgakaki@gmail.com
}

How to cite this paper: Georgakaki, S. K., \& Karakasidou, E. (2017). The Effects of Motivational Self-Talk on Competitive Anxiety and Self-Compassion: A Brief Training Program among Competitive Swimmers. Psychology, 8, 677-699.

https://doi.org/10.4236/psych.2017.85044

Received: February 11, 2017

Accepted: March 28, 2017

Published: March 31, 2017

Copyright $\odot 2017$ by authors and Scientific Research Publishing Inc. This work is licensed under the Creative Commons Attribution International License (CC BY 4.0).

http://creativecommons.org/licenses/by/4.0/

\begin{abstract}
The present study aimed to investigate the effects of motivational self-talk on competitive anxiety and self-compassion. Participants were 44 swimming athletes with competitive experience ranging in age from 18 to 25 . The experiment was conducted in five sessions. In the first session, the baseline assessment took place. Participants completed three questionnaires assessing the use of self-talk (Zervas, Stavrou, \& Psychountaki, 2007), competitive anxiety levels (Martens, 1977; Zervas \& Kakkos, 1990) and self-compassion levels (Neff, 2003b; Mantzios, Wilson, \& Giannou, 2013). After the baseline assessment participants were randomly divided and assigned as experimental $(N=$ 23) and control group $(N=21)$. Participants in the experimental group received a three-sessions training program on motivational self-talk in which they familiarized with its concept and its use. Participants in the control group did not receive any training. In the fifth session the final assessment took place in which the procedure of the baseline assessment was repeated. Results revealed that after the self-talk training program, participants in the experimental group indicated increments in the use of self-talk, as well as reduction in competitive anxiety levels and increases in self-compassion levels, whereas no changes were revealed for the control group. The findings of the study provided further support for the effectiveness of motivational self-talk strategies in generating desired affective states related with the participation in competitive sports. The results are discussed in relation to theory and related studies. Implications and suggestions for future research are also discussed.
\end{abstract}

\section{Keywords}

Self-Talk, Competitive Sport Anxiety, Self-Compassion, Training Program 


\section{Introduction}

It is well established that participation in physical activity and sports can enhance well-being and health (Lundqvist, 2011). However, competitive sports are very demanding and characterized by remarkable physical and psychological pressures. Furthermore, it has been indicated for many years that psychological factors play an important role in competitive sports and affect performance (Lizuka, 2005). Accordingly, high-level competitive athletes are faced with various difficulties concerning not only their performance but also their psychological well-being (Lundqvist, 2011).

Due to this fact, there are many training programs in which sport psychologists implement a variety of cognitive-behavioral strategies with the objective of aiding athletes in the development of psychological skills to enhance performance, manage emotions and achieve personal well-being (Ryska, 1998; Vealey, 2007). Current literature in applied sport psychology has indicated that cognitive-behavioral strategies are effective in generating a variety of desired affective states related with the participation in competitive sports (Ryska, 1998; Sheard \& Golby, 2006; Nazam \& Husain, 2014). One of the most common cognitivebehavioral strategies that have attracted researchers' attention in the last decades and used by athletes is "self-talk" (Hatzigeorgiadis, Theodorakis, \& Zourbanos, 2004).

\subsection{Self-Talk}

Hackfort \& Schwenkmezger (1993: p. 355) defined self-talk as an "inner speech in which the person interprets emotions and perceptions, manage and alter evaluations and cognitions and provide itself instructions and reinforcement". A more recent description of self-talk has been provided by Hardy, Hall, \& Hardy (2005: p. 905) and has been characterized as a "multidimensional phenomenon regarding athletes' verbalizations that are addressed to themselves". These selfstatements could represent "automatic verbalizations or more deliberate forms of speech and can be expressed aloud or as a silent voice in one's mind" (Hardy \& Oliver, 2014: p. 660).

Accordingly, self-talk has been categorized in two types in relation to its content-positive and negative self-talk (Theodorakis, Hatzigeorgiadis, \& Chroni, 2008). Positive self-talk has been described as an asset that increases self-esteem, motivation and concentration while negative self-talk considered to be critical, self-judgmental, anxiety producing and counterproductive (Johnson, Hrycaiko, Johnson, \& Halas, 2004; Hatzigeorgiadis, Theodorakis, \& Zourbanos, 2004). In reviewing the literature on the topic, field and laboratory-based research appeared to provide rather ambiguous results. Hardy, Gammage, and Hall (2001) suggested that support for the use of positive self-talk is stemming from experimentally-based research while field-based research has demonstrated mixed results. Van Raalte and colleagues, suggested that a plausible explanation for the mixed results was due to recall bias and they indicated that negative self-talk could possibly motivate some athletes (Van Raalte, Brewer, Rivera, \& Petitpas, 
1994). While Van Raalte and colleagues (1994) were the first to suggest that negative self-talk may be motivational for some athletes, Rushall (1984) nearly three decades ago suggested that self-talk could serve different functions for athletes including task-specific statements and positive mood words (as cited in Van Raalte et al., 1994).

Consequently, more recent approaches have discriminated positive self-talk into two broader dimensions-instructional and motivational self-talk (Zinsser, Bunker \& Williams, 2001, as cited in Hatzigeorgiadis et al., 2004). Instructional self-talk refers to self-statements associated with attentional focus (e.g., "see the target"), technical information (e.g., "high elbow"), and tactical choices (e.g., "push"), whereas motivational self-talk refers to self-statements associated with confidence building (e.g., "I can do it"), effort input (e.g., "give it all") and positive moods (e.g., "I feel good”) (Zourbanos, Hatzigeorgiadis, Bardas, \& Theodorakis, 2013; Hatzigeorgiadis, Zourbanos, \& Galanis, 2011). Research has demonstrated that different types of self-talk could serve different functions according to the content of self-talk (Hatzigeorgiadis, 2006). Therefore, different self-talk strategies could be implemented for different purposes. Instructional self-talk is primarily used for facilitating specific skills for the technical and tactical aspects of a sport while motivational self-talk is used to enhance psychological, mental and physical aspects of an athletes' well-being (Hatzigeorgiadis, 2006; Hatzigeorgiadis, Zourbanos, \& Theodorakis, 2007).

It is essential at this point to clarify that the current study was focused only on the (positive) motivational self-talk. As previously mentioned motivational selftalk is used primarily for enhancing athletes' well-being (Hatzigeorgiadis, 2006) who are confronted with tremendous pressures in order to achieve success in the sport setting (Lundqvist, 2011). One of the most common psychological issues encountered at the elite level is competitive anxiety.

\subsection{Competitive Sport Anxiety}

Martens, Burton, Vealey, Bump and Smith in 1990, developed the Multi-Dimensional Anxiety Theory which center of attention was primarily on competitive anxiety. They proposed that competitive anxiety is consisted of two sub-components, namely cognitive and somatic anxiety. Cognitive anxiety involves worries and negative thoughts about performance that preoccupy the individual, whereas somatic anxiety involves the physical symptoms that an individual may experience, such as tension, excessive sweating and stomach pain (Martens et al., 1990, as cited in Athan \& Sampson, 2013). Therefore, competitive anxiety refers to the cognitive and somatic anxiety than an athlete may experience in a competitive sport setting (Conroy \& Metzler, 2004). The theory also suggested that while somatic anxiety might diminish when athletes starts performing, the cognitive anxiety will remain if their self-confidence is low (Martens et al., 1990, as cited in Athan \& Sampson, 2013).

Cognitive theories of anxiety claim that automatic verbalizations and disturbing thoughts that occur in a continuously and rapid way during anxious states 
have been characterized as the primary dysfunction of anxiety (Conroy \& Metzler, 2004). Research studies in both sport and non-sport contexts has consistently related anxiety with negative self-talk (Hiebert, Uhlemann, Marshall, \& Lee, 1998; Kendall \& Treadwell, 2007; Hatzigeorgiadis \& Biddle, 2008). While dysfunctional thinking may not be problematic in some contexts, in a competitive sport environment it might have detrimental effects on performance (Birrer, Röthlin, \& Morgan, 2012; Humara, 1999). Restructuring the content of these thoughts and minimizing their frequency, forms the basis of motivational selftalk strategies to reducing competitive anxiety (Humara, 1999; Conroy \& Metzler, 2004; Singh, 2014).

\subsection{Motivational Self-Talk and Competitive Anxiety}

Research, adopting various designs, has indicated that self-talk strategies are effective in aiding athletes in the use of positive self-talk and consequently in reducing competitive anxiety levels (Conroy \& Metzler, 2004; Maynard, Smith, \& Warwick-Evans, 1995; Maynard, Hemmings, Greenlees, Warwick-Evans, \& Stanton, 1998; Hatzigeorgiadis, Zourbanos, \& Theodorakis, 2007; Hatzigeorgiadis et al., 2009). Owing to the fact that self-talk is not always an observable behavior, experimentally designed research employ manipulation checks, such as validated questionnaires, to document evidence that participants are engaging in the use of given instructions (Hatzigeorgiadis et al., 2009).

Preliminary evidence has been provided by Maynard et al. (1995) and Maynard et al. (1998) cognitive intervention studies, were positive thought control was implemented. Positive thought control involved the control of negative thoughts and their use in a positive way and training in how to replace negative thoughts with positive affirmations (Maynard et al., 1995; Maynard et al., 1998). Both studies indicated that positive thought control interventions were effective and resulted in reductions of competitive anxiety levels (Maynard et al., 1995; Maynard et al., 1998).

Similar findings have been provided by Hatzigeorgiadis, Zourbanos \& Theodorakis (2007) who examined the effects of self-talk on self-confidence, frequency of disturbing thoughts and competitive anxiety in 21 female swimmers. Results indicated that motivational self-talk was effective in reducing the frequency of disturbing thoughts and anxiety levels, as well as, in increasing selfconfidence (Hatzigeorgiadis et al., 2007). However, limitations of the study that should be consider were the absence of a control group and the fact that sample consisted only by females which cannot ensure the generalizability of results.

Moreover, Hatzigeorgiadis and colleagues (2009) conducted a study to examine the effectiveness of motivational self-talk training on self-confidence and competitive anxiety in tennis players. After completing three training sessions on the use of motivational self-talk, participants in the training group demonstrated that they made adequate use of the self-talk technique (Hatzigeorgiadis et al., 2009). Accordingly, the experimental group demonstrated increases in selfconfidence and reductions in competitive anxiety levels compared to the control 
group (Hatzigeorgiadis et al., 2009). Authors suggested that self-confidence was likely the underlying mechanism between self-talk and anxiety, suggesting that self-talk enhanced self-confidence leading in turn to reduced competitive anxiety (Hatzigeorgiadis et al., 2009). Results suggested that even a short training on motivational self-talk strategy could be beneficial not only for engaging athletes in the use of the technique, but also in generating the desired outcomes (Hatzigeorgiadis et al., 2009).

Overall, current literature indicated that motivational self-talk interventions are effective in generating a variety of desired affective states related with the participation in competitive sports and enhancing aspects of psychological wellbeing.

\subsection{Self-Compassion}

A recently new concept that has been linked with adaptive psychological functioning and aspects of psychological well-being, is self-compassion (Neff, Kirkpatrick, \& Rude, 2007; Smeets, Neff, Alberts, \& Peters, 2014). Self-compassion is simply compassion associated and addressed to ourselves, in a caring and concerning way, when we are faced with suffering experiences (Neff, 2003a). Neff (2003a) has defined self-compassion as being consisted of three main elements: "self-kindness versus self-judgment", "common humanity versus isolation", and "mindfulness versus over-identification". Self-kindness refers to the proclivity to be supportive and understanding with ourselves rather being critical and judgmental, especially in times of distress Neff (2003a). Common humanity involves acknowledging that every person can fail, make mistakes and face various life challenges and it is an important component of self-compassion as it helps the individual to see the self s imperfections from a broader perspective rather than feeling isolated Neff (2003a). Mindfulness entails being aware of one's suffering experiences in a clear and balanced way which neither avoids nor ruminates negative thoughts and emotions so that these thoughts and emotions are neither suppressed nor exaggerated Neff (2003a).

The psychological construct of self-compassion has received increased attention in the psychology field with a plurality of studies examining the influence of self-compassion on well-being (e.g., Hall, Row, Wuensch, \& Godley, 2013; Smeets et al., 2014). Research findings strongly supports positive relations between selfcompassion and various aspects of well-being, including mastery goals, life satisfaction, happiness, optimism, positive affect and personal initiative, and negative associations with sadness, negative affect, depression, anxiety, neuroticism, self-criticism, thought suppression and rumination (Neff, 2003a; Neff, 2003b; Neff, Kirkpatrick, \& Rude, 2007; Neely, Schallert, Mohammed, Roberts, \& Chen 2009). Recently, empirical evidence has also supported the association of selfcompassion with aspects of psychological well-being in the sports field (Mosewich, Kowalski, Sabiston, Sedgwick, \& Tracy, 2011; Mosewich, Crocker, Kowalski, \& Delongis, 2013; Ferguson, Kowalski, Mack, \& Sabiston, 2014; Reis, Kowalski, Ferguson, Sabiston, Sedgwick, \& Crocker, 2015). 
Mosewich and colleagues (2011) in a preliminary study attempted to examine self-compassion as a potential resource for adolescent female athletes. Their findings indicated self-compassion to be negatively related to guilt and shame proneness, social anxiety, body shame, fear of failure, and fear of negative evaluation (Mosewich et al., 2011). Results implied that self-compassion can act as a buffer against dysfunctional self-evaluative emotions, thoughts and behaviors (Mosewich et al., 2011); outcomes stemming from the various challenges athletes are faced with in their sport experiences.

More recently, Mosewich and colleagues (2013) in a randomized control trial, attempted to examine the effectiveness of a self-compassion intervention, which consisted of psychoeducational sessions and self-compassionate writing components, on negative cognitive states in women athletes who identified themselves as being self-critical. Their research indicated the effectiveness of the self-compassion intervention in enhancing self-compassion and managing self-criticism, rumination, and worries of making mistakes (Mosewich et al., 2013).

Similar findings have been reported by Ferguson and colleagues (2014) who used a mixed methods research design to examine self-compassion and wellbeing in young female athletes. Results from the quantitative study indicated that self-compassion was positively related with eudemonic well-being (Ferguson et al., 2014). Accordingly, findings from qualitative study described selfcompassion as a powerful tool for athletes when faced with difficult situations in the sport setting (Ferguson et al., 2014). Suffering from injuries, failing to achieve goals, performance plateauing and making mistakes during competition have been identified by participants as the most challenging situations that they can be faced with, and self-compassion appeared to help achieve athletes' potential by decreasing rumination, as well as by increasing positivity, responsibility and perseverance (Ferguson et al., 2014).

Consistent findings have been reported by Reis and colleagues (2015) who indicated that female athletes with higher self-compassion levels reacted, thought and felt in far less dysfunctional ways to emotionally difficult sport experiences than female athletes with lower self-compassion levels.

However, a common limitation of all studies previously mentioned is that selfcompassion has been explored only with female athletes. Therefore, results need to be replicated with samples being consisted of both males and females in order to ensure the generalizability of results.

Overall, the current literature suggested that self-compassion act as a protector against negative emotions, thoughts and behaviors that related with failure and negative events, suggesting that self-compassion should be considered in terms of potential application in the sport field as it can help athletes to cope with their challenging sport experiences (Mosewich et al., 2013; Reis et al., 2015). Considering that motivational self-talk training programs aim to teach participants to become aware of their negative thoughts and self-talk, it is therefore possible that motivational self-talk training could also be an effective way for individuals to develop self-compassion. Nonetheless, two more research studies 
have contributed to the development of this hypothesis.

Neff, Kirkpatrick and Rude (2007) conducted a study in order to investigate the relation of self-compassion to psychological well-being. They used a "Gestalt two-chair" technique which is an exercise that aims to decrease self-critical beliefs, and help people become more compassionate with themselves by giving voice in two conflicting aspects of self, namely self-critical voice and the "experiencing voice" which feels criticized and responds to the criticism (Greenberg, 1983; Safran, 1998, as cited in Neff, Kirkpatrick, \& Rude, 2007). Their findings indicated that increases in self-compassion levels were linked with increases in psychological well-being (Neff, Kirkpatrick, \& Rude, 2007). Specifically, results demonstrated that participants who shown increases in self-compassion also shown decreases in self-criticism, thought suppression, depression, and anxiety (Neff, Kirkpatrick, \& Rude, 2007). Furthermore, it was indicated that self-compassion acted as a protector against self-evaluative anxiety (Neff, Kirkpatrick, \& Rude, 2007). Further, reflecting on the technique they used, authors suggested that there are possibly many other techniques that could be used to increase self-compassion (Neff, Kirkpatrick, \& Rude, 2007).

Consequently, the first attempt to examine, indirectly though, the effects of self-talk on self-compassion was made by Al Awamleh, Mansi \& Ermeley (2014) who investigated the effect of a mental training skill program consisted of self-talk, goal setting, imagery and relaxation, on self-compassion in female college students. Their findings indicated that participation in the mental training program led to increases in self-compassion levels (Al Awamleh et al., 2014). However, a causal relationship between self-talk and self-compassion cannot be assumed by this study, as the researchers employed various techniques in the mental training program, thus it has not been established whereas self-talk or the combination of these techniques have influenced the results. Moreover, the several methodological limitations of the study cannot be overpassed. The sample consisted only of females, therefore it cannot ensure the generalizability of results. Moreover, the absence of control group along with the fact that the sample was not randomized may have introduced bias into the measurements.

\subsection{Study Aims}

In relation to this literature review, the present study aimed to replicate previous findings which suggested that motivational self-talk is an effective strategy in reducing competitive anxiety (Maynard et al., 1995; Maynard et al., 1998; Conroy \& Metzler, 2004; Hatzigeorgiadis et al., 2007; Hatzigeorgiadis et al., 2009). Furthermore, taking into account the work of Neff, Kirkpatrick, \& Rude (2007) and Al Awamleh et al. (2014), as well as considering the suggestion of Neff, Kirkpatrick, \& Rude (2007) that possibly other techniques could also be able to increase self-compassion, the present study attempted to examine the effectiveness of motivational self-talk intervention on enhancing self-compassion levels in athletes with competitive experience.

Overall, the study aimed to examine the effects of a motivational self-talk 
training program on aspects of psychological well-being in competitive swimmers. Specifically, the study aimed to indicate that after the motivational selftalk training program, the Self-Talk Intervention Group would demonstrate increments in the use of self-talk and secondary that the Self-Talk Intervention Group would demonstrate lower levels of competitive anxiety and higher levels of self-compassion compared to the Control Group.

\section{Methodology}

\subsection{Design}

A 2 (Group: STQ Intervention vs. Control Group) $\times 2$ (Time: Pretest vs. Posttest) mixed design ANOVA with Group as a between-subjects factor and Time as a within-subjects factor was used in this study, to examine whether motivational self-talk reduces competitive anxiety and enhances self-compassion. Three measurements were taken at each time; the scores achieved from participants' self-reported use of self-talk, competitive anxiety and self-compassion.

\subsection{Participants}

The sample consisted of 44 Greek swimmers who were recruited through opportunity sampling and volunteered for the study. Participants were members of the Sport Nautical Club of Argyroupolis (swimming club in Athens) who had competitive swimming experience and had not undertaken any training in the use of self-talk. In all, 23 were males and 21 were females, ranging in age from 18 to 25 , with mean age 19.8 years $(S D=2.16)$ and mean competitive experience 10.6 years $(S D=2.99)$. Participants were randomly assigned as experimental ( $n$ $=23)$ and control $(n=21)$ group. All participants were treated according to the ethical standards of the British Psychological Society.

\subsection{Instruments}

Self-Talk Questionnaire ([S-TQ]; Zervas, Stavrou, \& Psychountaki, 2007). The questionnaire consists of 11 statements marked in a Likert scale (from $1=$ never to 5 = always) and measures two forms of ST function (motivational and cognitive) that used by athletes to enhance their sport performances. Seven of the eleven statements assess the motivational functions (e.g., "I talk to myself to increase my effort") and the four assess the cognitive functions (e.g., "I talk to myself to give directions"). The instrument also provides a total score of self-talk use, ranging from 11 (very low self-talk use) to 55 (very high self-talk use). Zervas et al. (2007) reported high internal consistency coefficients of .91 for the motivational and .84 for the cognitive self-talk. The Cronbach's a for the total S-TQ was .92 (Zervas et al., 2007). The Cronbach's alpha for the current study was .91 and .94 at pre and post training respectively.

Sport Competition Anxiety Test ([SCAT]; Martens, 1977; Zervas \& Kakkos, 1990). The Greek version of the SCAT was used that has been translated into Greek by Zervas \& Kakkos (1990). The questionnaire consists of 10 items 
marked in a 3-point Likert scale ( $1=$ hardly ever, $2=$ sometimes, $3=$ often $)$ and assess the levels of competitive trait anxiety of athletes. More specifically, SCAT consists of two statements assessing the anxiety factor (e.g., "before I compete I feel uneasy") two statements assessing the calmness factor (e.g., "before I compete I am calm") and six statements assessing the psychosomatic factor (e.g., "Just before I compete I notice that my heart beats faster than normal"). The questionnaire provides a total score of competitive trait anxiety with scores ranging from 10 to 30 . Lower scores reflect a lower tendency to confront with competitive anxiety and higher scores indicate a higher tendency to experience competitive anxiety. The original test demonstrated high internal consistency ranging from .95 to .97 (Martens, 1977). The Greek version of SCAT reported a range of .84 to .89 (Zervas \& Kakkos, 1990). The Cronbach's alpha for the present study ranged from .85 to .87 .

Self-Compassion Scale ([SCS]; Neff, 2003b; Mantzios, Wilson, \& Giannou, 2013). The scale consists of 26 items marked in a 5-point Likert scale ranging from $1=$ almost never to $5=$ almost always). It is composed of six subscales that includes the 5-item self-kindness subscale (e.g., "I'm kind to myself when I'm experiencing suffering"), the 5-item self-judgement subscale (e.g. "When times are really difficult, I tend to be tough on myself"), the 4-item common humanity subscale (e.g., "I try to see my failings as part of the human condition"), the 4-item isolation subscale (e.g., "When I fail at something that's important to me, I tend to feel alone in my failure"), the 4-item mindfulness subscale (e.g., "When I fail at something important to me I try to keep things in perspective") and the 4-item over-identification subscale (e.g., "When something upsets me I get carried away with my feelings"). The instrument provides an overall self-compassion score by averaging mean scores of the six subscales (after reverse-coding negative items), with overall scores ranging from 26 to 130 . Self-Compassion Scale was used in its Greek version that has been translated in Greek by Mantzios et al. (2013). The original scale demonstrated excellent reliability ( $\alpha=.93$; e.g., Neff, 2003b). Accordingly, Mantzios et al. (2013) reported internal consistency coefficients of .87. The Cronbach's alpha for the present study was .82 and .91 at pre and post training respectively.

\section{Demographic Information}

Participants were asked to fill out a demographic questionnaire related to age, gender, favored swimming strokes, swimming achievements and years of competitive experience.

\subsection{Procedure}

Permission to conduct the study was obtained by the ICPS committee associated with University of Central Lancashire (UCLAN), which is a committee whose task it is to make sure that research participants are protected from harm. In order to gain access to the Sport Nautical Club, coaches and sport psychologist had to be informed about the nature of study and give permission. The experiment was completed in five sessions, which took place once a week with duration of 90 
minutes.

\section{Session 1: Baseline assessment}

Participants were approached and asked to participate voluntarily in a research aiming to examine athletes' psychological well-being. After informing them about confidentiality issues and their right to withdraw from the study at any time and any stage the initial assessment took place. Participants were administered a booklet containing a consent form and the above-mentioned questionnaires, namely the demographic information, the Self-Talk Questionnaire, the Sport Competition Anxiety Test and the Self-Compassion Scale in different order to control for order effect. No names or other identification was required, thus maintaining anonymity. The booklet took approximately 15 minutes to complete.

After the completion of the baseline assessment, participants were randomly divided into two groups and assigned as experimental and control. Experimental group was informed that the current study had a psycho-educational objective that aimed to train athletes with competitive experience to develop their psychological skills in order to enhance aspects of their psychological well-being. They were further informed that if they were still willing to participate, the program would require them to attend in three more meetings of 90 minutes in which they would be trained based on basic theories of sport psychology. Accordingly, the control group was also informed that the study would require a second meeting in four weeks in which the initial assessment would be repeated. Both groups were informed again about confidentiality issues and their right to withdraw from the study at any time and any stage and that in the case of withdrawn their data will be automatically deleted and will not be used for any other purpose.

\section{Session 2 - 4: Training phase}

The planning and implementation of the program was built upon the work of Mamassis \& Doganis (2004) and Hatzigeorgiadis, Zourbanos, Goltsios \& Theodorakis (2008). At the beginning of the second session the experimental group was introduced in the concept of self-talk. In short, they were told that self-talk is the talk we have with ourselves in which we evaluate what we have done and how we feel about it. In sessions that were followed participants were taught the importance of self-talk and its connection to negative thoughts and emotions as well as its link with low performance. The first step for them was to become aware of their negative thoughts and statements before, during or after a swimming meet. Subsequently, participants were trained and taught to stop the negative by using key words (e.g., "stop", "park it") and replace the negative thoughts with positive ones by using motivational cues ("I can", "Let's go", "I've got this"). The final step for them was to apply the techniques they have learned during practice. The training program was applied to groups of 7 or 8 athletes. The control group was not given any training.

\section{Session 5: Final Assessment}

On the fifth session the procedure of the initial assessment was repeated. After 
the completion of the assessment participants were debriefed, explained the purpose of the study and thanked for their participation.

\section{Results}

For the aims of this study, participants' data that were collected through the questionnaires were analyzed (See the attached SPSS file for raw data). The means and standard deviations of self-talk, competitive anxiety and self-compassion scores for all participants at pre-test measurements were analyzed. Moreover, the correlations between these variables were calculated in order to check for inter-correlations and presented in Table 1.

A two-tailed Pearson's correlation revealed a medium, negative and significant correlation between self-talk and competitive anxiety scores $(r=-.32, p$ $=.034)$. No significant correlations were reported neither between self-talk and self-compassion scores $(r=.16, p=.289)$ either between competitive anxiety and self-compassion scores $(r=-.29, p=.054)$.

Accordingly, the Table 2 below presented the mean scores and standard deviations for pre-test and post-test measures for intervention group and control group separately.

A series of One-Way independent samples ANOVAs was employed to examine whether there were significant differences between the self-talk intervention group and control group on self-talk, competitive anxiety and self-compassion measures at baseline assessment. ANOVAs revealed no significant differences

Table 1. Mean scores, SDs and bivariate correlations between variables at pre-test $(N=$ 44).

\begin{tabular}{cccc}
\hline & Self-Talk & Competitive Anxiety & Self-Compassion \\
\hline Self-Talk & -- & $-.32^{*}$ & .16 \\
Competitive Anxiety & -- & -.29 \\
Self-Compassion & & -- \\
$M$ & 31.36 & 23.61 & 65.84 \\
$S D$ & 9.23 & 4.18 & 10.9 \\
\hline
\end{tabular}

Note. ${ }^{*} p<.05$.

Table 2. Means and SDs of all study variables at pre-test and post-test measurements for intervention and control group.

\begin{tabular}{ccccccccc}
\hline & \multicolumn{3}{c}{ STQ Intervention Group $(N=23)$} & \multicolumn{3}{c}{ Control Group $(N=21)$} \\
\cline { 2 - 8 } & \multicolumn{2}{c}{ Pre-test } & \multicolumn{2}{c}{ Post-test } & \multicolumn{2}{c}{ Pre-test } & \multicolumn{2}{c}{ Post-test } \\
\cline { 2 - 8 } & \multicolumn{2}{c}{$S D$} & $M$ & $S D$ & $M$ & $S D$ & $M$ & $S D$ \\
\hline STQ & 33.44 & 9.78 & 43.44 & 6.10 & 29.09 & 8.23 & 28.71 & 7.38 \\
CA & 23.22 & 3.67 & 20.39 & 4.04 & 24.05 & 4.73 & 23.81 & 3.92 \\
SC & 67.82 & 9.78 & 77.74 & 10.76 & 63.67 & 11.88 & 63.00 & 12.74 \\
\hline
\end{tabular}

${ }^{*}$ Note: STQ $=$ Self-Talk, CA = Competitive Anxiety, SC = Self-Compassion. 
between groups on any of the study measures at the baseline assessment. Specifically, no significant differences were found between groups on self-talk scores $[F(1,42)=2.51, p=.121]$, competitive anxiety scores $[F(1,42)=.43, p=.517]$ and self-compassion scores $[F(1,42)=1.62, p=.210]$.

Accordingly, a series of paired samples $t$-tests was conducted to examine prepost changes in study measures for each group separately.

Two-tailed paired samples $t$-tests revealed that there was a significant increase in self-talk scores $[t(22)=-8.16, p<.001]$, in self-compassion scores $[t(22)=$ $-4.91, p<.001]$, as well as a significant reduction in competitive anxiety scores for all participants of the intervention group.

Additionally, two-tailed paired samples $t$-tests revealed that there were no significant differences in self-talk scores $[t(20)=1.14, p=.268]$, competitive anxiety scores $[t(20)=.50, p=.624]$, and self-compassion scores $[t(20)=1.70, p$ $=.105]$ for participants of the control group.

Next, a series of 2(Group: STQ Intervention vs. Control Group) $\times 2$ (Time: Pretest vs. Posttest) mixed design ANOVAs with Group as a between-subjects factor and Time as a within-subjects factor were employed in order to determine whether STQ Intervention Group indicated larger improvements compared to the Control Group, as measured by the scores achieved from participants' responds in self-talk use, competitive anxiety and self-compassion measures.

\subsection{Self-Talk Use}

There was a significant main effect of Time on self-talk use, $[F(1,42)=52.86, p$ $<.001], \mathrm{Eta}^{2}=.56(56 \%)$. Examination of the marginal means (See Figure 1 below) indicated that self-talk use was higher at the post-test measurement.

A significant main effect was also found for Group, $[F(1,42)=16.86, p<.001]$, $\operatorname{Eta}^{2}=.29$ (29\%). Examination of the marginal means (See Figure 1) indicated that self-talk use was higher in the STQ Intervention Group and lower in the Control Group.

Moreover, a significant interaction between Group and Time was also found $[F(1,42)=61.57, p<.001], \mathrm{Eta}^{2}=.59(59 \%)$, indicating that the effect of Time depended upon the Group. The 95\% CI graph of the interaction (see Figure 2 below) indicated that differences between pre and post self-talk use measures were least for the Control Group and greatest for the STQ Intervention Group.

A two-tailed independent samples t-test demonstrated that there was a significant difference between STQ Intervention Group and Control Group in the post-test measurement. The STQ Intervention Group presented higher scores in the use of self-talk compared to the Control Group [ $t(42)=7.23, p<.001]$. Levene's test indicated that homogeneity of variance assumptions were met $(F=$ $2.235, p=.142)$

\subsection{Competitive Anxiety}

There was a significant main effect of Time on competitive anxiety scores, $[F(1,42)=11.60, p=.001], \mathrm{Eta}^{2}=.22(22 \%)$. Examination of the marginal means 


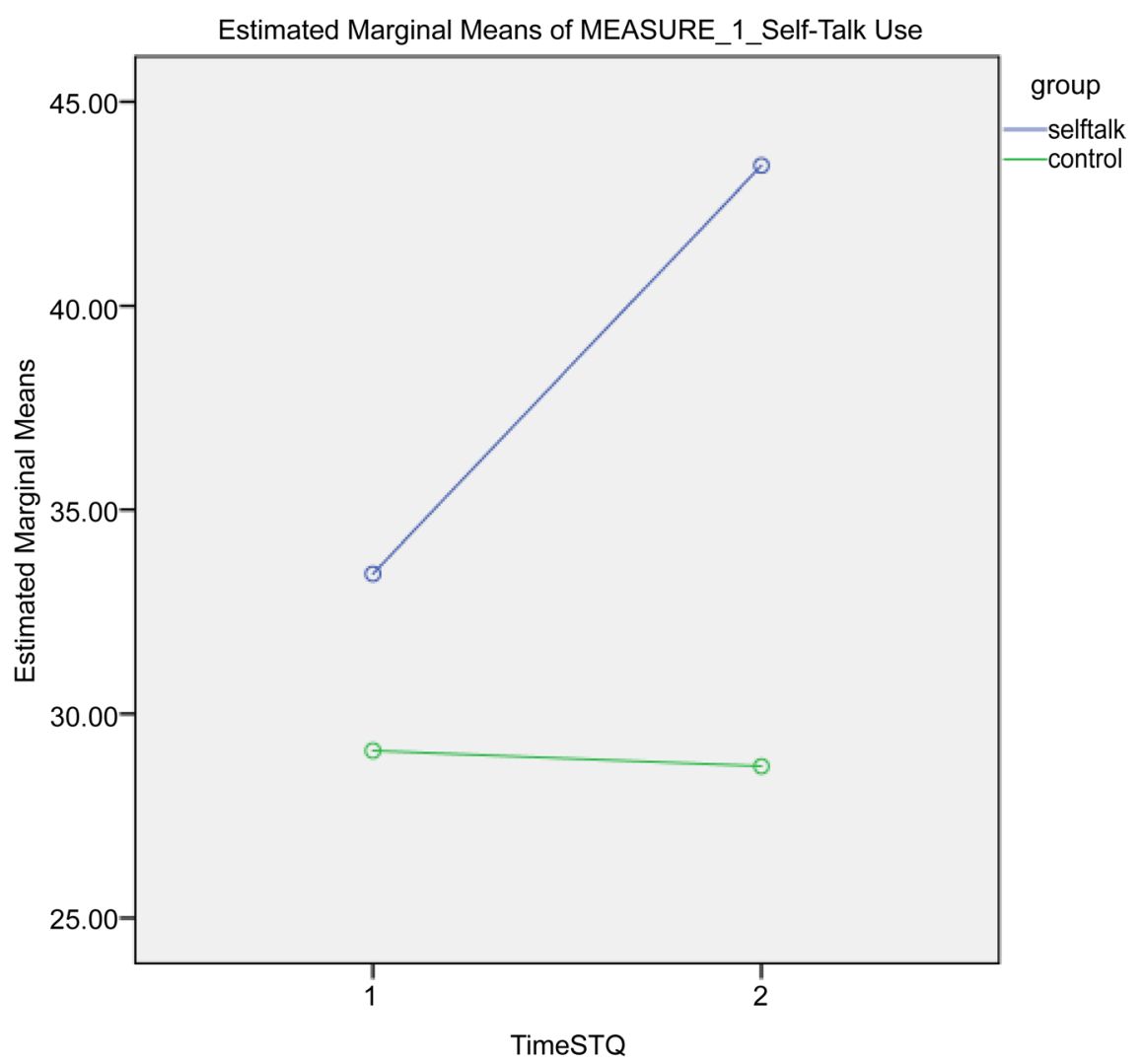

Figure 1. Estimated marginal means for self-talk use.

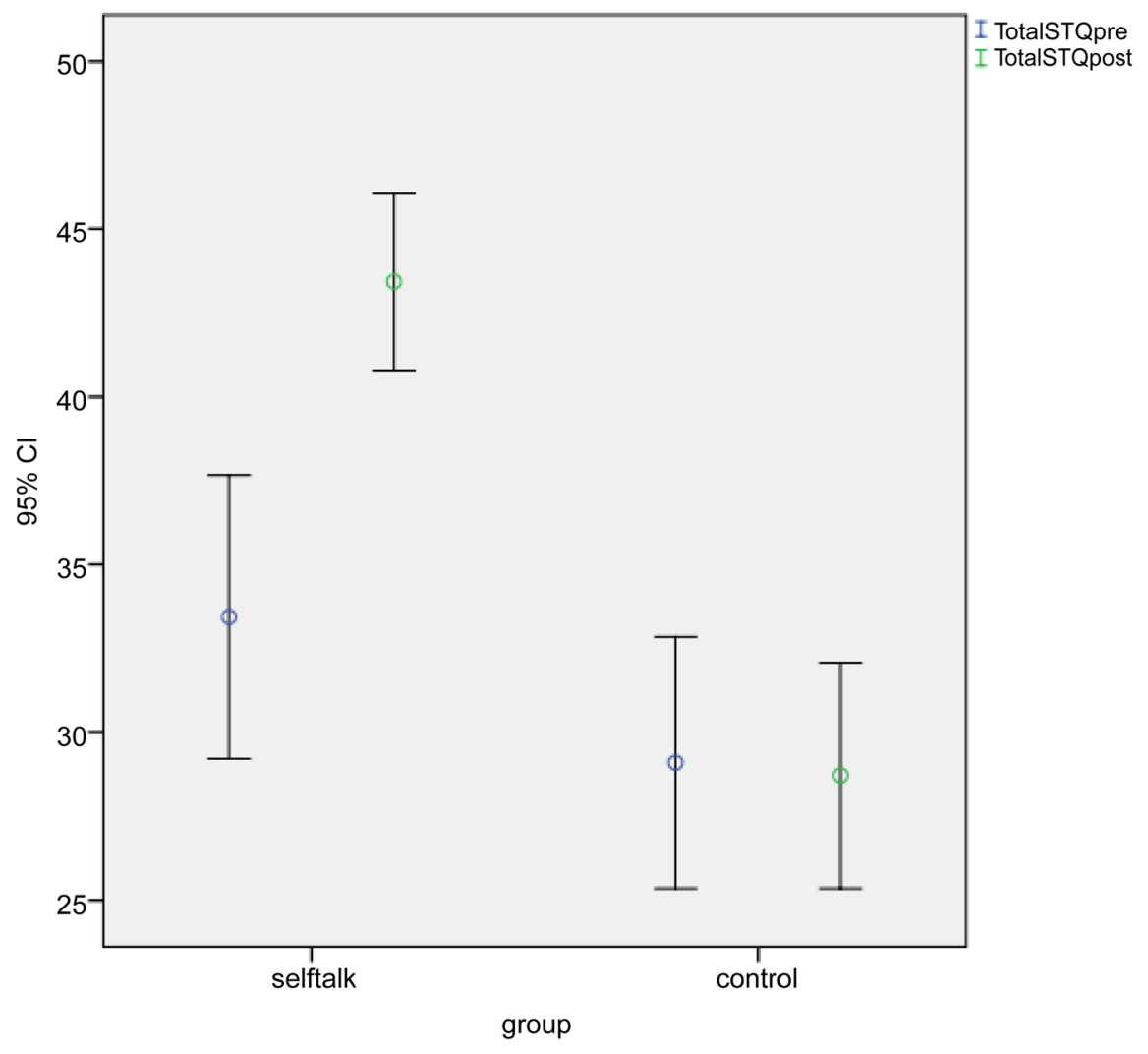

Figure 2. 95\% CI graph of the interaction between group and time. 
(See Figure 3 below) indicated that competitive anxiety levels were lower at the post-test measurement.

However, no significant main effect of Group was observed, $[F(1,42)=3.40, p$ $=.07], \mathrm{Eta}^{2}=.08(8 \%)$.

Finally, a significant interaction between Group and Time was found $[F(1,42)$ $=8.28, p=.006], \mathrm{Eta}^{2}=.17(17 \%)$, indicating that the effect of Time depended upon the Group. The 95\% CI graph of the interaction (see Figure 4) indicated that differences between pre and post competitive anxiety measures were least for the Control Group and greatest for the STQ Intervention Group.

A two-tailed independent samples t-test demonstrated that there was a significant difference between STQ Intervention Group and Control Group in the post-test measurement. The STQ Intervention Group presented lower scores in competitive anxiety levels compared to the Control Group $[t(42)=-2.84, p$ $=.007]$. Levene's test indicated that homogeneity of variance assumptions were met $(F=1.503, p=.227)$.

\subsection{Self-Compassion}

There was a significant main effect of Time on self-compassion levels, $[F(1,42)=$ $18.50, p<.001], \mathrm{Eta}^{2}=.31(31 \%)$. Examination of the marginal means (See Figure 5) indicated that self-compassion levels were higher at post-test measurement.

A significant main effect was also found for Group, $[F(1,42)=8.53, p=.006]$, $\mathrm{Eta}^{2}=.17(17 \%)$. Examination of the marginal means (See Figure 5) indicated

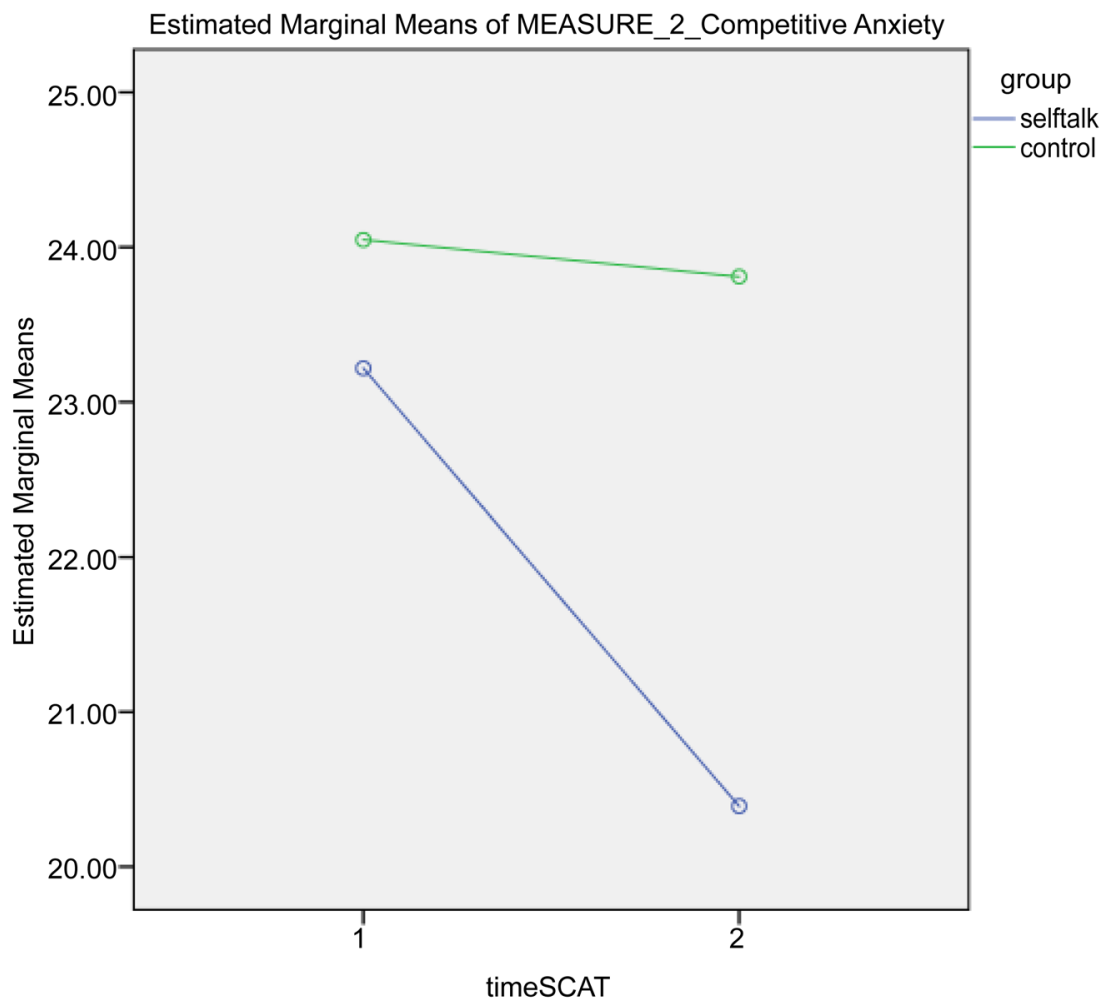

Figure 3. Estimated marginal means for competitive anxiety. 


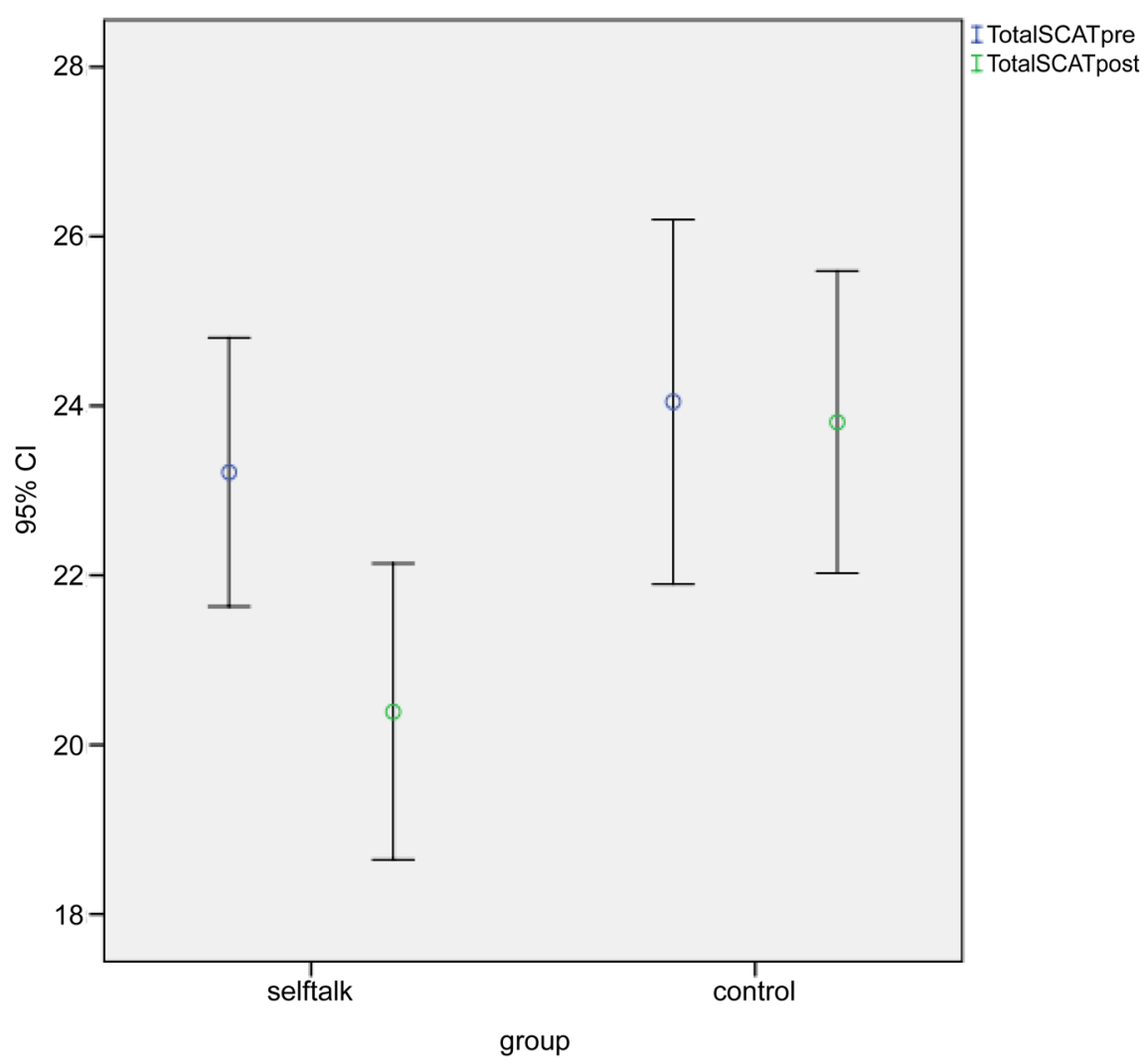

Figure 4. 95\% CI graph of the interaction between group and time.

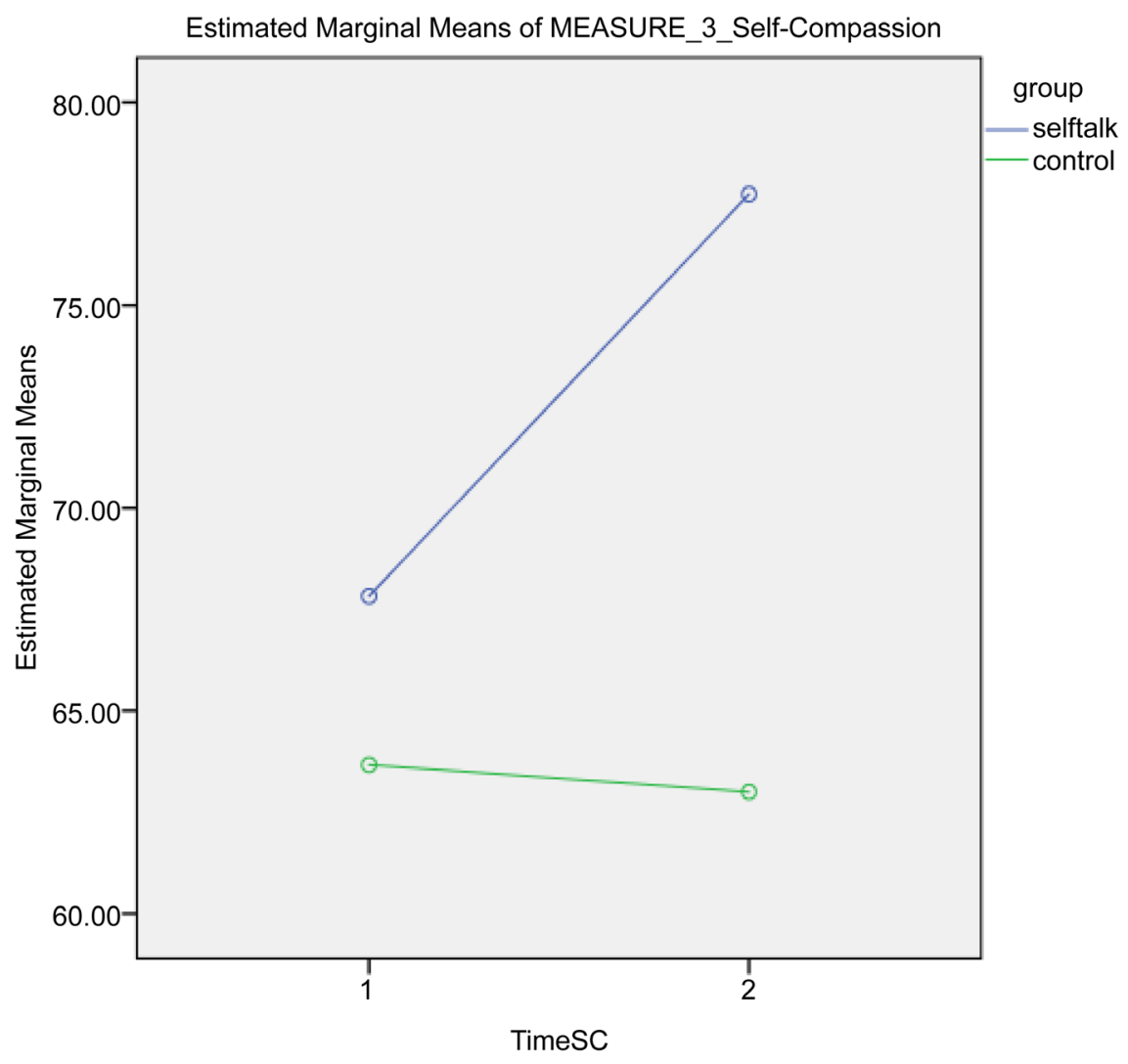

Figure 5. Estimated marginal means for self-compassion. 
that self-compassion levels were higher in the STQ Intervention Group and lower in Control Group.

Moreover, a significant interaction between Group and Time was also found $[F(1,42)=24.22, p<.001], \mathrm{Eta}^{2}=.37(37 \%)$, indicating that the effect of Time depended upon the Group. The 95\% CI graph of the interaction (see Figure 6 below) indicated that differences between pre and post self-compassion measures were least for the Control Group and greatest for the STQ Intervention Group.

A two-tailed independent samples t-test demonstrated that there was a significant difference between STQ Intervention Group and Control Group in the post-test measurement. The STQ Intervention Group presented higher scores in self-compassion levels compared to the Control Group [ $t(42)=4.16, p<.001]$. Levene's test indicated that homogeneity of variance assumptions were met ( $F$ $=.935, p=.339$ ).

\section{Discussion}

The present study aimed to examine the effects of a motivational self-talk training program on aspects of psychological well-being in competitive swimmers. Specifically, the study aimed to demonstrate that after the self-talk training program, participants in the Intervention Group would indicate increments in the use of self-talk, as well as lower levels of competitive anxiety and higher levels of self-compassion compared to the Control Group. Overall, results indicated that

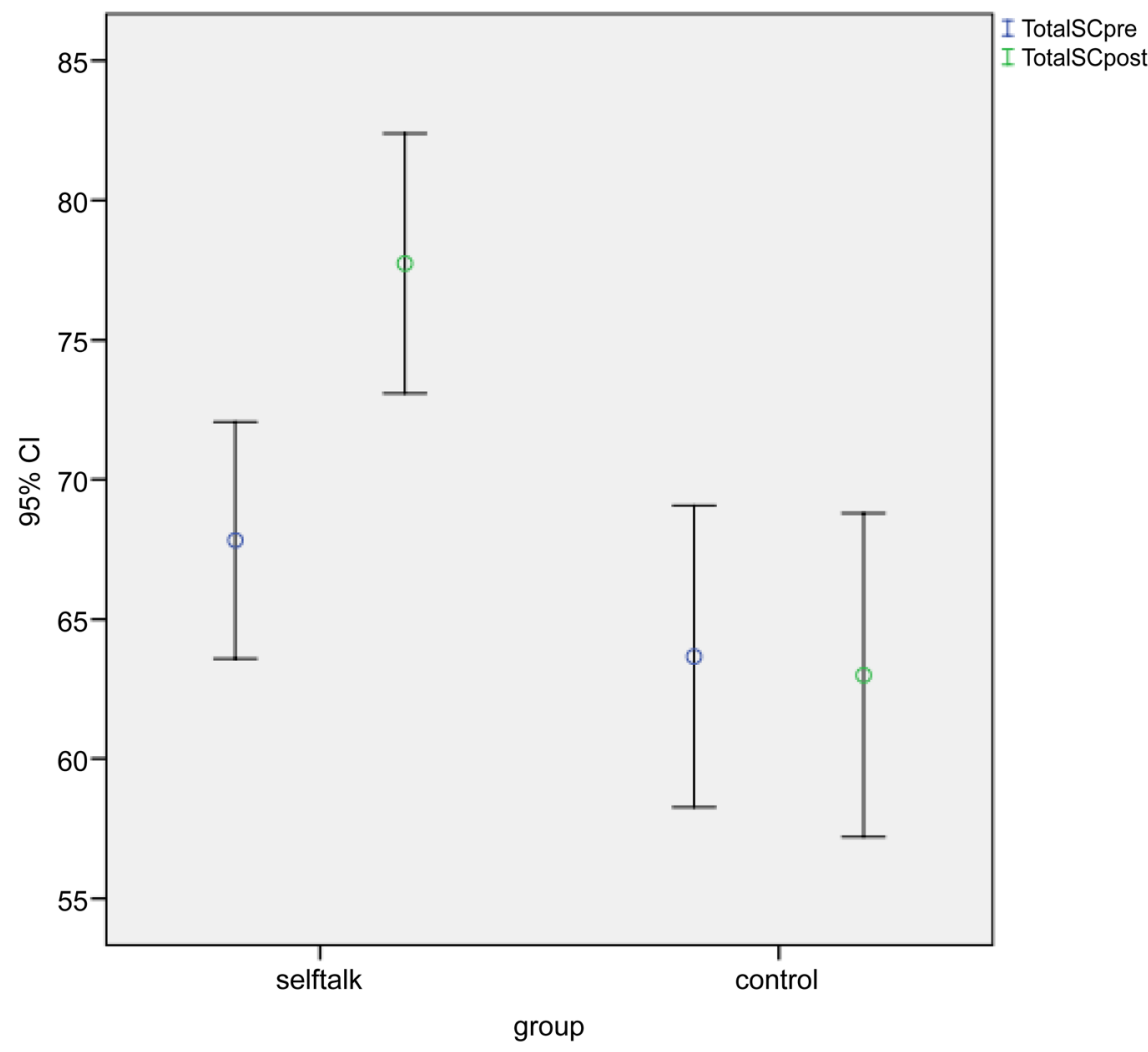

Figure 6. 95\% CI graph of the interaction between group and time. 
self-talk training program had a positive effect on self-talk use, reduced competitive anxiety and increased self-compassion.

Prior to proceeding to the hypotheses testing, participants in the intervention group received the appropriate motivational self-talk training. Briefly, they were trained to become aware of their negative thoughts and statements, replace those thoughts and statements with more positive-motivational ones and familiarized with the appropriate use of self-talk through practice. Because as Conroy \& Metzler (2004) and Singh (2014) suggested, the basis of motivational self-talk strategies to generating desired outcomes relies on restructuring the content of negative thoughts and minimizing their frequency. Findings regarding the use of self-talk in the first assessment and final assessment, indicated that participants in the intervention group demonstrated significant increases in the use of selftalk compared to control group. Results from the study supported previous evidence which suggested that self-talk strategies are effective in aiding athletes in the use of positive self-talk (Maynard et al., 1995; Maynard et al., 1998; Hatzigeorgiadis, Zourbanos, \& Theodorakis, 2007; Hatzigeorgiadis et al., 2009). Specifically, Maynard and colleagues $(1995,1998)$ as well as Hatzigeorgiadis, Zourbanos \& Theodorakis (2007) indicated that controlling negative thoughts and replacing them with positive affirmations were effective in reducing the frequency of disturbing thoughts and engaging in the use of motivational self-talk. Furthermore, findings from the present study, suggested that even a short training on motivational self-talk was beneficial for engaging athletes in the use of the technique as Hatzigeorgiadis et al. (2009) previously pointed out.

Moreover, with regard to the competitive anxiety, results from this study showed that participants in the intervention group demonstrated reductions in competitive anxiety levels compared to participants in the control group, suggesting that self-talk training was effective in reducing competitive anxiety. Results are consistent with previous findings reported by Maynard et al. (1995, 1998) who indicated that positive thought control interventions were effective and resulted in reductions of competitive anxiety levels. Results also validated the findings reported by Hatzigeorgiadis, Zourbanos \& Theodorakis (2007) which showed that after receiving motivational self-talk training participants demonstrated reduction in the frequency of disturbing thoughts, as well as, reduction in competitive anxiety levels.

According to the Multi-Dimensional Anxiety Theory, cognitive anxiety arises from worries and negative thoughts that preoccupy individuals, and that cognitive anxiety may depend on their self-perceptions of confidence (Martens et al., 1990, as cited in Athan \& Sampson, 2013). Therefore, while motivational selftalk is effective in reducing the frequency of negative and disturbing thoughts (Hatzigeorgiadis, Zourbanos, \& Theodorakis, 2007), it is consequently effective in reducing anxiety levels. Furthermore, as motivational self-talk involves selfstatements associated with confidence building (Zourbanos et al., 2013) it is plausible that self-confidence could be one of the underlying mechanisms between self-talk and anxiety. Confirmatory findings have been reported by Hatzi- 
georgiadis et al. (2009) who indicated that motivational self-talk training was effective in increasing self-confidence and reducing competitive anxiety levels. Authors suggested that self-talk enhanced self-confidence leading in turn to reduced competitive anxiety (Hatzigeorgiadis et al., 2009).

Additionally, another important aim of this study was that it attempted, drawing on the work of Neff, Kirkpatrick, \& Rude (2007) and Al Awamleh et al. (2014), to investigate the effectiveness of motivational self-talk training on enhancing self-compassion levels. Results indicated that participants who received the motivational self-talk training, demonstrated increases in self-compassion levels from the first assessment to the final assessment compared to participants in control group. Findings suggested that motivational self-talk training is an effective strategy that can enhance self-compassion in participants, supporting thus the suggestion of Neff, Kirkpatrick, \& Rude (2007) that possibly many techniques could be used to increase self-compassion levels.

Although, self-compassion has never been examined as a potential outcome of self-talk interventions in the sports domain, results could be interpreted in relation to the study of Neff, Kirkpatrick, \& Rude (2007) which investigated the relation of self-compassion to psychological well-being. More specific, Neff and colleagues (2007), used the "Gestalt two-chair" technique, as a method for increasing self-compassion levels in undergraduate students. The technique's main objective was to decrease self-critical beliefs, and thus help people become more compassionate with themselves (Neff, Kirkpatrick, \& Rude, 2007). Their findings indicated that their method was effective in increasing self-compassion levels, and that participants who shown increases in self-compassion also shown decreases in self-criticism (Neff, Kirkpatrick, \& Rude, 2007). Considering that the "Gestalt two-chair" technique aimed to decrease self-critical beliefs, and that motivational self-talk strategies rely among others on minimizing the frequency of negative self-talk, which considered to be critical and self-judgmental (Hatzigeorgiadis, Theodorakis, \& Zourbanos, 2004; Johnson et al., 2004), has provided the rationale behind comparing the current study with the study of Neff, Kirkpatrick, \& Rude (2007). Furthermore, the findings of the present study could also be compared with the study of Al Awamleh et al. (2014) who investigated the effect of a mental training skill program consisted of self-talk, goal setting, imagery and relaxation, on self-compassion, and found that participation in the mental training program led to increases in self-compassion levels in female college students.

Empirical evidence has emphasized the importance of self-compassion in female athletes. In particular, previous research has indicated that self-compassion can act as a buffer against dysfunctional self-evaluative emotions, thoughts and behaviors (Mosewich et al., 2011). Furthermore, Reis and colleagues (2015) demonstrated that female athletes with higher self-compassion levels reacted, thought and felt in far less dysfunctional ways to emotionally difficult sport experiences than female athletes with lower self-compassion levels. Thus, the development of self-compassion could be a powerful tool for athletes when faced 
with difficult situations and psychological pressures in the sport setting (Ferguson et al., 2014), because it could help them cope with all these challenging experiences and potentially to enhance their performance (Mosewich et al., 2013; Reis et al., 2015).

Taking findings into consideration, the present study has both theoretical and practical implications. From a theoretical angle current findings expand existing knowledge regarding the effects of motivational self-talk. While the study replicated previous findings which suggest that motivational self-talk is effective in reducing competitive anxiety (e.g. Maynard et al., 1998; Hatzigeorgiadis et al., 2009) nevertheless, its major contribution was that motivational self-talk can also enhance self-compassion. From a practical prospect, the study highlights the importance of motivational self-talk in regulating competitive anxiety and enhancing self-compassion. Therefore, athletes themselves, coaches and sport psychologists are encouraged to incorporate in their strategies and further advance the use of motivational self-talk, in order to manage emotions, improve psychological well-being and enhance performance (Vealey, 2007; Sheard \& Golby, 2006). Finally, given the short duration of the training program and its effectiveness in generating positive outcomes, could enable researchers, as well as coaches and sport psychologists, to apply these types of training more often, since brief trainings and studies are less time consuming and less expensive.

It is important at this point to acknowledge some research limitations. First, the current study recruited participants that consisted only by swimming athletes. Although, many studies have been conducted while recruiting participants from a single sport category, nonetheless it is important that studies should be done among other sports as well, so as to ensure the generalizability of results.

Furthermore, another limitation that should be acknowledged is that the study was based exclusively on self-report data. Although this is evident to most other studies in this area, nonetheless socially desirable responding might have introduced bias in the results. Future research should administer measures of social desirability in order to aid control for this potential bias, as well as, behavioral measures to strengthen self-reported outcomes.

In addition, considering that the assessment of constructs was concurrent, the issue of causation could not be resolved. Future research could address this issue with longitudinal designs. Additionally, another limitation of the study was that it did not include follow-up measures and assessments and therefore the lasting effects of the intervention cannot yet be verified. Still, the duration and preservation of intervention effects and therapeutic gains need to be examined in future research.

A last issue that should be addressed has to do with the lack of desired statistical power in some of the comparative analysis that were conducted, probably due to the sample size. However, owing to the fact that this was an intervention study, the sample size can be considered satisfactory. Further research is needed to extend the findings and the accuracy of the current results. An important future goal would also be to expand research by investigating the various subcom- 
ponents of self-compassion so as to examine how they may have deferentially been affected by self-talk, since the present study only examined the overall score of self-compassion.

\section{Conclusion}

In conclusion, findings of the present study indicated that motivational self-talk training program had a positive effect on self-talk use, reduced competitive anxiety levels and increased self-compassion levels. The current study highlights the importance of motivational self-talk training in regulating performance variables, such as competitive anxiety and self-compassion. It is important to create and implement training programs, not only to enhance performance, which is the major concern of competitive athletes, but also to enhance their psychological well-being. Athletes themselves, as well as sport psychologists and coaches, owe to protect athletes' mental and psychological health, because in essence, these programs that enhance their psychological well-being, are the ones that will increase their commitment and will enable them through the pleasure they derive from what they do, to improve their performance.

\section{References}

Al Awamleh, A., Mansi, T., \& Ermeley, Z. (2014). The Effect of Mental Training Skills Program on Self-Compassion and Mindfulness. Asian Social Science, 10, 90-95. https://doi.org/10.5539/ass.v10n18p90

Athan, A. N., \& Sampson, U. I. (2013). Coping with Pre-Competitive Anxiety in Sports Competition. European Journal of Natural and Applied Sciences, 1, 1-9.

Birrer, D., Röthlin, P., \& Morgan, G. (2012). Mindfulness to Enhance Athletic Performance: Theoretical Considerations and Possible Impact Mechanisms. Mindfulness, 3, 235-246. https://doi.org/10.1007/s12671-012-0109-2

Conroy, D. E., \& Metzler, J. N. (2004). Patterns of Self-Talk Associated with Different Forms of Competitive Anxiety. Journal of Sport \& Exercise Psychology, 26, 69-89. https://doi.org/10.1123/jsep.26.1.69

Ferguson, L. J., Kowalski, K. C., Mack, D. E., \& Sabiston, C. M. (2014). Exploring Self-Compassion and Eudaimonic Well-Being in Young Women Athletes. Journal of Sport \& Exercise Psychology, 36, 203-216. https://doi.org/10.1123/jsep.2013-0096

Hackfort, D., \& Schwenkmezger, P. (1993). Anxiety. In R. N. Singer, M. Murphy, \& L. K. Tennant (Eds.), Handbook of Research on Sport Psychology (pp. 328-364). New York: Macmillan.

Hall, C. W., Row, K. A., Wuensch, K. L., \& Godley, K. R. (2013). The Role of Self-Compassion in Physical and Psychological Well-Being. The Journal of Psychology, 147, 311323. https://doi.org/10.1080/00223980.2012.693138

Hardy, J., \& Oliver, E. J. (2014). Self-Talk, Positive Thinking, and Thought Stopping. In T. Gershon, \& R. C. Eklund (Eds.), Encyclopedia of Sport and Exercise Psychology. Thousand Oaks, CA: Sage.

Hardy, J., Gammage, K., \& Hall, C. R. (2001). A Descriptive Study of Athletes Self-Talk. The Sport Psychologist, 15, 306-318. https://doi.org/10.1123/tsp.15.3.306

Hardy, J., Hall, C. R., \& Hardy, L. (2005). Quantifying Athlete Self-Talk. Journal of Sports Sciences, 23, 905-917. https://doi.org/10.1080/02640410500130706

Hatzigeorgiadis, A. (2006). Instructional and Motivational Self-Talk: An Investigation on 
Perceived Self-Talk Functions. Hellenic Journal of Psychology, 3, 164-175.

Hatzigeorgiadis, A., \& Biddle, S. J. (2008). Negative Self-Talk during Sport Performance: Relationships with Pre-Competition Anxiety and Goal-Performance Discrepancies. Journal of Sport Behavior, 31.

http://www.biomedsearch.com/article/Negative-self-talk-during-sport/183423084.html

Hatzigeorgiadis, A., Theodorakis, Y., \& Zourbanos, N. (2004). Self-Talk in the Swimming Pool: The Effects of Self-Talk on Thought Content and Performance on Water-Polo Tasks. Journal of Applied Sport Psychology, 16, 138-150. https://doi.org/10.1080/10413200490437886

Hatzigeorgiadis, A., Zourbanos, N., \& Galanis, E. (2011). Self-Talk and Sports Performance: A Meta-Analysis. Perspectives on Psychological Science, 6, 348-356. https://doi.org/10.1177/1745691611413136

Hatzigeorgiadis, A., Zourbanos, N., \& Theodorakis, Y. (2007). The Moderating Effects of Self-Talk Content on Self-Talk Functions. Journal of Applied Sport Psychology, 19, 240-251. https://doi.org/10.1080/10413200701230621

Hatzigeorgiadis, A., Zourbanos, N., Goltsios, C., \& Theodorakis, Y. (2008). Investigating the Functions of Self-Talk: The Effects of Motivational Self-Talk on Self-Efficacy and Performance in Young Tennis Players. The Sport Psychologist, 22, 458-471.

https://doi.org/10.1123/tsp.22.4.458

Hatzigeorgiadis, A., Zourbanos, N., Mpoumpaki, S., \& Theodorakis, Y. (2009). Mechanisms Underlying the Self-Talk-Performance Relationship: The Effects of Motivational Self-Talk on Self-Confidence and Anxiety. Psychology of Sport and Exercise, 10, 186192.

Hiebert, B., Uhlemann, M. R., Marshall, A., \& Lee, D. Y. (1998). The Relationship Between Self-Talk, Anxiety, and Counselling Skill. Canadian Journal of Counselling, 32, 163-171.

Humara, M. (1999). The Relationship between Anxiety and Performance: A Cognitive-Behavioral Perspective. The Online Journal of Sport Psychology, 1, 1-14.

Johnson, J. J. M., Hrycaiko, D. W., Johnson, G. V., \& Halas, J. M. (2004). Self-Talk and Female Youth Soccer Performance. The Sport Psychologist, 18, 44-59. https://doi.org/10.1123/tsp.18.1.44

Kendall, P. C., \& Treadwell, K. R. H. (2007). The Role of Self-Statements as a Mediator in Treatment for Youth with Anxiety Disorders. Journal of Consulting and Clinical Psychology, 75, 380-389. https://doi.org/10.1037/0022-006X.75.3.380

Lizuka, P. (2005). Anxiety and Performance in Young Table Tennis Players. Sports Science Research, 26, 73-75.

Lundqvist, C. (2011). Well-Being in Competitive Sports-The Feel-Good Factor? A Review of Conceptual Considerations of Well-Being. International Review of Sport and Exercise Psychology, 4, 109-127. https://doi.org/10.1080/1750984X.2011.584067

Mamassis, G., \& Doganis, G. (2004). The Effects of a Mental Training Program on Juniors Pre-Competitive Anxiety, Self-Confidence, and Tennis Performance. Journal of Applied Sport Psychology, 16, 118-137. https://doi.org/10.1080/10413200490437903

Mantzios, M., Wilson, J. C., \& Giannou, K. (2013). Psychometric Properties of the Greek Versions of the Self-Compassion and Mindful Attention and Awareness Scales. Mindfulness, 6, 123-132. https://doi.org/10.1007/s12671-013-0237-3

Martens, R. (1977). Sport Competition Anxiety Test. Champaign, IL: Human Kinetics.

Maynard, I. W., Hemmings, B., Greenlees, I. A., Warwick-Evans, L., \& Stanton, N. (1998). Stress Management in Sport: A Comparison of Unimodal and Multimodal Interventions. Anxiety, Stress, \& Coping, 11, 225-246. 
https://doi.org/10.1080/10615809808248313

Maynard, I. W., Smith, M. J., \& Warwick-Evans, L. (1995). The Effects of a Cognitive Intervention Strategy on Competitive State Anxiety and Performance in Semiprofessional Soccer Players. Journal of Sport \& Exercise Psychology, 17, 428-446. https://doi.org/10.1123/jsep.17.4.428

Mosewich, A. D., Crocker, P. R. E., Kowalski, K. C., \& Delongis, A. (2013). Applying Self-Compassion in Sport: An Intervention with Women Athletes. Journal of Sport \& Exercise Psychology, 35, 514-524. https://doi.org/10.1123/jsep.35.5.514

Mosewich, A. D., Kowalski, K. C., Sabiston, C. M., Sedgwick, W. A., \& Tracy, J. L. (2011). Self-Compassion: A Potential Resource for Young Women Athletes. Journal of Sport \& Exercise Psychology, 33, 103-123. https://doi.org/10.1123/jsep.33.1.103

Nazam, F., \& Husain, A. (2014). Enhancing Sports and Exercise Performance through Cognitive Interventions. Indian Journal of Positive Psychology, 5, 28-32.

Neely, M. E., Schallert, D. L., Mohammed, S. S., Roberts, R. M., \& Chen, Y. (2009). Self-Kindness When Facing Stress: The Role of Self-Compassion, Goal Regulation, and Support in College Students Well-Being. Motivation and Emotion, 33, 88-97. https://doi.org/10.1007/s11031-008-9119-8

Neff, K. D. (2003a). Self-Compassion: An Alternative Conceptualization of a Healthy Attitude toward Oneself. Self and Identity, 2, 85-101. https://doi.org/10.1080/15298860309032

Neff, K. D. (2003b). The Development and Validation of a Scale to Measure Self Compassion. Self and Identity, 2, 223-250. https://doi.org/10.1080/15298860309027

Neff, K. D., Kirkpatrick, K. L., \& Rude, S. S. (2007). Self-Compassion and Adaptive Psychological Functioning. Journal of Research in Personality, 41, 139-154.

Reis, N. A., Kowalski, K. C., Ferguson, L. J., Sabiston, C. M., Sedgwick, W. A., \& Crocker, P. R. E. (2015). Self-Compassion and Women Athletes' Responses to Emotionally Difficult Sport Situations: An Evaluation of a Brief Induction. Psychology of Sport \& Exercise, 16, 18-25.

Ryska, T. A. (1998). Cognitive-Behavioral Strategies and Precompetitive Anxiety among Recreational Athletes. The Psychological Record, 48, 697-708.

Sheard, M., \& Golby, J. (2006). Effect of a Psychological Skills Training Program on Swimming Performance and Positive Psychological Development. International Journal of Sport and Exercise Psychology, 4, 149-169. https://doi.org/10.1080/1612197X.2006.9671790

Singh, R. (2014). Personality, Spiritual Exercise and Cognitive-Behavioral Interventions in Enhancing Sports Performance. Indian Journal of Positive Psychology, 5, 301-309.

Smeets, E., Neff, K., Alberts, H., \& Peters, M. (2014). Meeting Suffering with Kindness: Effects of a Brief Self-Compassion Intervention for Female College Students. Journal of Clinical Psychology, 70, 794-807.

Theodorakis, Y., Hatzigeorgiadis, A., \& Chroni, S. (2008). Self-Talk: It Works, but How? Development and Preliminary Validation of the Functions of Self-Talk Questionnaire. Measurement in Physical Education and Exercise Science, 12, 10-30. https://doi.org/10.1080/10913670701715158

Van Raalte, J. L., Brewer, B. W., Rivera, P. M., \& Petitpas, A. J. (1994). The Relationship between Observable Self-Talk and Competitive Junior Tennis Players' Match Performances. Journal of Sport and Exercise Psychology, 16, 400-415. https://doi.org/10.1123/jsep.16.4.400

Vealey, R. S. (2007). Mental Skills Training in Sport. In T. Gershon, \& R. C. Eklund (Eds.), Handbook of Sport Psychology (3rd ed., pp. 285-309). Hoboken, NJ: John Wiley \& 
Sons, Inc. https://doi.org/10.1002/9781118270011.ch13

Zervas, J., \& Kakkos, V. (1990). Validity and Reliability of Sport Competition Anxiety test in Greek Population. Sport Psychology, 4, 3-24.

Zervas, Y., Stavrou, N., \& Psychountaki, M. (2007). Development and Validation of the Self-Talk Questionnaire (S-TQ) for Sports Journal of Applied Sport Psychology, 19, 142-159.

Zourbanos, N., Hatzigeorgiadis, A., Bardas, D., \& Theodorakis, Y. (2013). The Effects of a Self-Talk Intervention on Elementary Students' Motor Task Performance. Early Child Development and Care, 183, 924-930. https://doi.org/10.1080/03004430.2012.693487

Submit or recommend next manuscript to SCIRP and we will provide best service for you:

Accepting pre-submission inquiries through Email, Facebook, LinkedIn, Twitter, etc. A wide selection of journals (inclusive of 9 subjects, more than 200 journals)

Providing 24-hour high-quality service

User-friendly online submission system

Fair and swift peer-review system

Efficient typesetting and proofreading procedure

Display of the result of downloads and visits, as well as the number of cited articles Maximum dissemination of your research work

Submit your manuscript at: http://papersubmission.scirp.org/

Or contact psych@scirp.org 